\title{
Radiative and inner-shell dielectronic recombination in a highly charged barium ion
}

\author{
D. J. McLaughlin \\ Department of Physics, University of Hartford, West Hartford, Connecticut 06117 \\ Y. Hahn \\ Department of Physics, University of Connecticut, Storrs, Connecticut 06269 \\ E. Takács, E. S. Meyer, and J. D. Gillaspy \\ Physics Laboratory, National Institute of Standards and Technology, Gaithersburg, Maryland 20899
}

(Received 28 September 1995)

\begin{abstract}
The direct radiative recombination (RR) to $n=3,4$, and 5 levels and the resonant dielectronic recombination (DR) cross section involving $2 p \rightarrow 3 d$ excitation of Sc-like barium $\mathrm{Ba}^{35+}$ and Ti-like barium $\mathrm{Ba}^{34+}$ are calculated and compared with a recent electron beam ion trap experiment at the National Institute of Standards and Technology. Assuming a fractional population of $\mathrm{Ba}^{35+}$ in the trap of about $30 \%$, we obtain good agreement between theory and experiment for the cross section ratio $\sigma(\mathrm{DR}) / \sigma(\mathrm{RR})(n=4)$, as well as for $\sigma(\mathrm{RR})(n=5) / \sigma(\mathrm{RR})(n=4)$. The result confirms again that the simple angular momentum averaged procedure can be effective in treating DR for heavy open-shell ions when the energy resolution is not high. A large, broad peak below $2 \mathrm{keV}$ in the $\mathrm{x}$-ray spectrum is being theoretically examined. [S1050-2947(96)04008-5]
\end{abstract}

PACS number(s): $34.80 . \mathrm{Kw}, 32.80 . \mathrm{Hd}$

\section{INTRODUCTION}

The availability of the electron beam ion trap (EBIT) at Lawrence Livermore National Laboratory (LLNL), Oxford, and the National Institute of Standards and Technology (NIST) has opened up a rich field of experiments with highly charged ions at low velocities. There have been several recent measurements of x-ray spectra emitted by highly charged ions produced by EBIT [1,2] and merged beams [3] that involve electron capture with inner-shell excitations.

In a recent investigation carried out with the EBIT at NIST, scandiumlike barium $\mathrm{Ba}^{35+}\left([\mathrm{Ar}] 3 d^{3}\right)$ and titaniumlike barium $\mathrm{Ba}^{34+}\left([\mathrm{Ar}] 3 d^{4}\right)$ ions were created, trapped, and excited using an electron beam of approximately $2.3 \mathrm{keV}$ energy. A strong x-ray emission peak was observed at $4.6 \mathrm{keV}$, and several smaller peaks were measured at lower energies, down to approximately $2 \mathrm{keV}$ where the spectrum is gradually cut off due to detector limitations (Fig. 1). This experiment is particularly interesting because x-ray peaks arising from both dielectronic recombination (DR), labeled $a$ in Fig. 1 , and radiative recombination (RR), labeled $b$ in Fig. 1, can be studied simultaneously. Since the binding energy of the $3 d$ orbital is about $2.3 \mathrm{keV}$ and noting the energy difference between the $2 p$ and $3 d$ orbitals is $4.6 \mathrm{keV}$, we attributed the principal $x$-ray peak to a DR process in which a continuum electron collisionally excites a $2 p$ electron in the trapped ion and is in turn captured into the $3 d$ orbital. One of the $M$-shell electrons ( $3 d$ ) of the recombined ion subsequently decays radiatively to the $2 p$ vacancy, thereby emitting a 4.6 $\mathrm{keV} x$-ray, i.e., an $\mathrm{x}$ ray of energy twice the incident kinetic energy of the projectile electron. The peak due to this DR process is denoted in Fig. 1 as $a b$ because it also contains contributions from RR corresponding to radiative capture into states with principal quantum number $n=3$. The smaller DR peak labeled $a^{\prime}$ at $4.0 \mathrm{keV}$ corresponds to radiative decay of an $M$-shell $3 s$ electron into the $2 p$ vacancy. The x ray peaks at $3.7 \mathrm{keV}(b 4)$ and at $3.3 \mathrm{keV}(b 5)$ and $3.4 \mathrm{keV}(b 4)$ correspond to direct radiative capture into states with $n=4$ and 5 , respectively, while the broad peak $(b)$ at $2.9 \mathrm{keV}$ corresponds to RR into states with $n \geqslant 6$. Finally, a very large and broad bump $(c)$ was observed, stretching over the energy range $1-2 \mathrm{keV}$. This peak is not treated here. The dependence of this spectrum on electron-beam energy was studied by varying the beam energy by as much as $100 \mathrm{eV}$ on both sides of the DR resonance. These spectra are shown in Fig. 2. We note that as the kinetic energy of the electron beam moves away from the DR resonance energy the RR peak in $a b$ shifts to higher energy while the resonances, $a$ and $a^{\prime}$, gradually disappear. Peaks $b 4$ and $b 5$ (as well as bump $c$ ) remain more or less the same in magnitude but

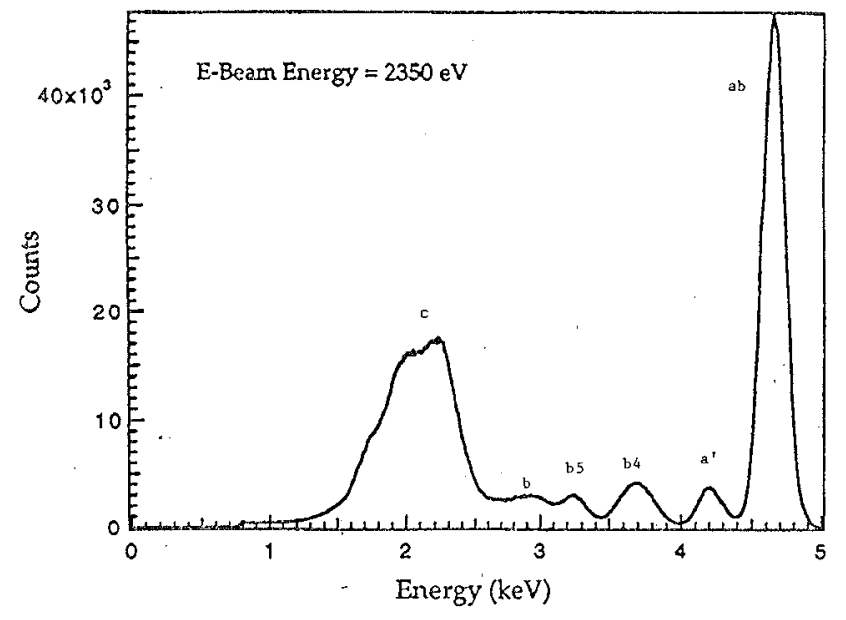

FIG. 1. Sc-like barium spectrum at an electron-beam energy of approximately $2350 \mathrm{eV}$ and a beam current of $65 \mathrm{~mA}$. The detector resolution is about $200 \mathrm{eV}$, and the spread in electron-beam energy is about $30 \mathrm{eV}$. The spectrum was acquired over six hours. 

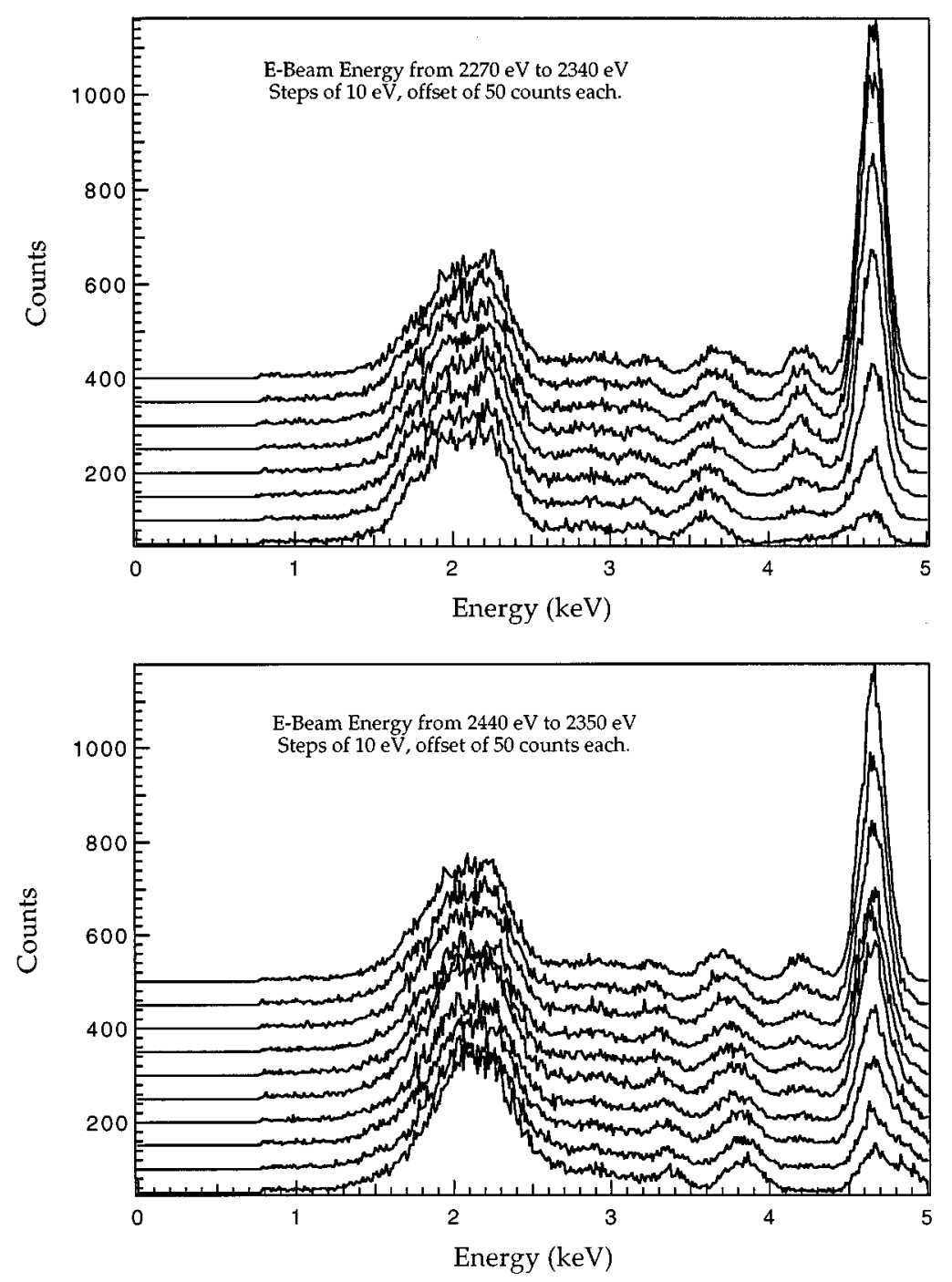

FIG. 2. Sc-like barium spectra taken at electron-beam energies ranging from $2270 \mathrm{eV}$ to $2440 \mathrm{eV}$ in steps of $10 \mathrm{eV}$. The top graph shows the spectra for energies above the main DR resonance, with the beam energy decreasing with offset from the $x$ axis. The bottom graph shows the spectra for energies below the main DR resonance, with the beam energy increasing with offset from the $x$ axis. slowly shift to higher energies with increasing electron energy. The main peak $a b$ is reduced in size off resonance, but there is a small recognizable peak which persists at about 4.6 $\mathrm{keV}$. This important information, together with approximate energy levels of the ground and excited states of $\mathrm{Ba}^{35+}$ calculated by Kim [4], is sufficient to assign tentatively the peaks $b 4$ and $b 5$ and the residual peak in $a b$ to RR processes, and the main portion of the peak $a b$ and $a^{\prime}$ to DR processes.

We present here the experimental data of DR and RR for the $\mathrm{Ba}^{34+}$ and $\mathrm{Ba}^{35+}$ target ions and details of an estimation of their cross sections. Our calculations indicate that the 4.6 $\mathrm{keV}$ peak $a b$ and the peak $a^{\prime}$ at $4 \mathrm{keV}$ do indeed arise from DR and estimates of the relative magnitude of the accompanying RR cross sections are found to be in favorable agreement with the data.

\section{EXPERIMENTAL DISCUSSION}

The operation of the EBIT has been described in several reviews [5]. Briefly, the EBIT consists of a 10-150 mA electron beam focused to less than $100 \mu \mathrm{m}$ diameter by a strong magnetic field. The electron beam is accelerated to between $1 \mathrm{keV}$ and $30 \mathrm{keV}$ by a series of positively biased cylindrical electrodes or drift tubes. Atoms and low-charged ions that are injected into the center of the drift tubes are stripped to higher-charge states by the electron beam and trapped radially by the space charge of the electron beam as well as the action of the magnetic field. The trap along the electron beam is formed by a collection of three drift tubes, a center drift tube and two end caps. The end caps are biased 100$500 \mathrm{~V}$ above the center one to provide axial trapping for the ions. The electron-ion interaction energy is controlled by the absolute voltage applied to the center drift tube.

Barium is perhaps the easiest element to study in the EBIT because it is a primary dopant in the electron gun cathode, and sufficient barium evaporates from the heated cathode to provide an abundant source for the trap. To make a specific charge state, the electron-beam energy must be tuned appropriately (to an energy above the ionization potential of the next lowest-charge state, but slightly below the ionization potential of the desired charge state). The energy of the electron beam is controlled by the voltage $V_{0}$ applied to the center drift tube, but it is not exactly equal to $e V_{0}$. The space charge of the electron beam depresses the on-axis potential somewhat, and therefore lowers the electron-beam energy below this. It is a simple matter to calculate the effect of the electron space charge on the energy [6], but inside the EBIT there is a complication due to the partial neutralization 
of the negative electron space charge by the positive charge of trapped ions. It is difficult to determine the total number of trapped ions of all charge states (including those of any background gas trapped), but through a combination of calculation and measurement of the position of certain radiative recombination (RR) lines, it is possible to get a good estimate. We estimate that the overall space-charge correction to the energy is $150 \mathrm{eV}$, with an uncertainty of $\pm 50 \mathrm{eV}$. In order to produce $\mathrm{Ba}^{35+}$, it is necessary for the electron-beam energy to be greater than the ionization potential of Ti-like barium $(2.259 \mathrm{keV})$. In order to prevent the conversion of $\mathrm{Ba}^{35+}$ into $\mathrm{Ba}^{36+}$, it is necessary to tune the beam energy to just below the ionization potential of the $\mathrm{Ba}^{35+}$ state itself (about $2.355 \mathrm{keV}$ ). We adjusted the drift tube potential so as to give a space-charge corrected value of the beam energy of about $2.35 \mathrm{keV}$. We confirmed that the beam energy was in the correct place by monitoring a previously observed [7] visible line in Ti-like barium using a monochromator and phototube. By maximizing the strength of this line and then increasing the center drift tube voltage by $100 \mathrm{~V}$, we could be confident that the beam was optimized for producing Sclike barium.

The EBIT was designed with several side ports at $90^{\circ}$ to the electron beam which look directly into the ion trap. Certain ports are covered by thin $(0.125 \mathrm{~mm})$ beryllium windows, so as to hold the vacuum without appreciably attenuating $\mathrm{x}$ rays of energy greater than $2.5 \mathrm{keV}$. We took a number of Sc-like barium spectra using a solid-state $\mathrm{Si}(\mathrm{Li})$ detector. The spectrum shown in Fig. 1 was integrated over six hours at an estimated beam energy of $2.35 \mathrm{keV}$ and a beam current of $56 \mathrm{~mA}$. Although the monochromaticity of the electron beam has not been measured directly, we expect that the energy spread in the beam is less than $50 \mathrm{eV}$ and probably less than $30 \mathrm{eV}$ full width at half maximum (FWHM). The detector has an energy resolution of approximately $200 \mathrm{eV}$, and the combined detector-window system has about $45 \%$ efficiency at $2.5 \mathrm{keV}$, which increases to about $75 \%$ at $3.5 \mathrm{keV}$ and $90 \%$ at $4.5 \mathrm{keV}$.

Using a computer simulation of the evolution of the charge state balance within the trap [8], which neglects the effect of DR, we estimate that over $90 \%$ of the trapped barium ions are either in the $\mathrm{Ba}^{34+}$ or $\mathrm{Ba}^{35+}$ states, with a ratio of 1:1.4 between them at $2.35 \mathrm{keV}$ electron-beam energy. Including the effect of the expected DR process in the calculation, the ratio of $\mathrm{Ba}^{35+}$ to $\mathrm{Ba}^{34+}$ may be reduced significantly. In the analysis that follows, we account for this reduction by allowing $P$, the ratio of $\mathrm{Ba}^{35+}$ ion density to the sum of the $\mathrm{Ba}^{35+}$ and $\mathrm{Ba}^{34+}$ ion densities, to be reduced by a factor of $\beta(0<\beta<1)$ which we track throughout the analysis to insure that the final comparison with theory is not circular. To confirm the line identifications described above, we measured spectra at a number of different beam energies in 10 $\mathrm{eV}$ steps. The precision with which we can change the center electron-beam energy is better than $2 \mathrm{eV}$, although the spread in electron-beam energy might be several tens of $\mathrm{eV}$. The spectra taken at steps in beam energy are shown in Fig. 2. The main peak $(a b)$ is reduced in magnitude as the beam energy is varied and at the same time its centroid shifts with energy. This is because the peak $(a b)$ is composed of one DR peak and one RR peak. The resonant DR peak does not move, but its intensity is reduced when the RR peak moves.
Therefore the overall shift is more apparant off resonance. The radiative recombination (RR) lines all shift in the direction of change in the beam energy.

\section{DR CROSS SECTION}

The DR process proceeds as

$$
e^{-}+A^{Z+}(i) \rightarrow A^{(Z-1)+* *}(d) \rightarrow A^{(Z-1)+*}(f)+\hbar \omega .
$$

The initial capture is a resonant process with conservation of energy and momentum leading to a doubly excited autoionizing resonance state $A^{* *}$. As this state relaxes by x-ray emission $(d \rightarrow f)$, the $\mathrm{x}$-ray energies $\hbar \omega=E_{d}-E_{f}$ provide the distinct signature of the process. The DR cross section $\sigma^{\mathrm{DR}}(i \rightarrow d \rightarrow f)$ calculated in the distorted-wave-projection operator method [9] and in the isolated resonance approximation, is given explicitly by

$$
\begin{aligned}
\sigma^{\mathrm{DR}}\left(i, e_{c} \rightarrow\right. & d \rightarrow f)=\frac{4 \pi}{\left(k_{c} a_{0}\right)^{2}} \tau_{0} V_{a}\left(d \rightarrow i, e_{c}\right) \omega(d \rightarrow f) \\
& \times \widetilde{\delta}\left(E_{d} \rightarrow E_{i}+e_{c}\right)\left(\pi a_{0}^{2}\right),
\end{aligned}
$$

where $k_{c}$ is the wave number of the continuum electron, $a_{0}$ is the Bohr radius, and $\tau_{0}$ is the atomic unit of time. $V_{a}(i \rightarrow d)$ is the inverse of the autoionization rate $A_{a}(d \rightarrow i)$ of the intermediate state, and they are related by $V_{a}=g_{d} /\left(2 g_{i}\right) A_{a}$, where $g_{d}$ and $g_{i}$ are the statistical weights of the intermediate and initial states, respectively. Also introduced in (2) is the fluorescence yield

$$
\omega(d)=\frac{\Gamma_{r}(d)}{\Gamma_{r}(d)+\Gamma_{a}(d)}=\frac{\Gamma_{r}(d)}{\Gamma(d)}=\sum_{f} \omega(d \rightarrow f),
$$

where $\Gamma_{r}(d)=\sum_{f} A_{r}(d \rightarrow f)$ and $\Gamma_{a}(d)=\sum_{i^{\prime}} A_{a}\left(d \rightarrow i^{\prime}\right)$ are the total radiative and autoionization widths, respectively, of the $d$ state of full width $\Gamma(d)=\Gamma_{r}(d)+\Gamma_{a}(d)$. The Lorentzian factor is defined as

$$
\begin{array}{r}
\widetilde{\delta}\left(E_{d} \rightarrow E_{i}+e_{c}\right) \equiv \frac{\Gamma(d)}{2 \pi\left[\left(E_{i}+e_{c}-E_{d}\right)^{2}-\frac{\Gamma(d)^{2}}{4}\right]}, \\
\text { with } \int \widetilde{\delta} d e_{c}=1 .
\end{array}
$$

All possible states $i$ that are connected to $d$, and which are allowed by energy conservation and angular momentum and parity rules, are summed over in $\Gamma_{a}(d)$.

The bound-state orbitals used in the calculation of $A_{a}(d \rightarrow i)$ and the radiative decay rate $A_{r}(d \rightarrow f)$ are computed numerically with the nonrelativistic, singleconfiguration Hartree-Fock code [10]. The continuum-wave function is calculated with the Hartree-Fock direct and explicit nonlocal exchange potentials. Because of the strong configuration mixing required for the ions of interest here, and the large number of angular momentum coupled states involved in the intermediate states $(d), A_{a}$ and $A_{r}$ may be conveniently evaluated in an angular momentum average (AMA) scheme in which all the couplings are averaged. This scheme only requires specification of the orbitals directly 
involved and their statistical factors [11]. Spectator electrons play no direct role in AMA, except to screen the active electrons (thereby altering the radial wave functions) and to modify some of the statistical factors. An additional justification for the choice of the AMA is that the experimental width of the electron beam is $\Delta B_{\exp } \sim 30$ to $50 \mathrm{eV}$, which averages out contributions from many term levels. Our past experience shows that the AMA and the resulting $\vec{\sigma}^{\mathrm{DR}}$ are quite reasonable, particularly when dominant states $d$ are involved, as is the case here [12]. [For a given $d$-state configuration there is only one AMA state but, in general, many $L S$ terms, so the calculation of DR cross sections is conveniently done in the AMA scheme. In fact, in the limit that $\Gamma_{r} \gg \Gamma_{a}$, $\omega(d) \sim 1$, and the total DR cross sections obtained in different coupling schemes are identical to that of the AMA because of the sum over all terms involved.] The AMA scheme tends to overestimate the cross section by roughly $20 \% \sim 40 \%$, however.

Since the full width $\Gamma(d)$ of the DR peak described by $\widetilde{\delta}$ is very narrow, on the order of $\sim 0.1 \mathrm{eV}$ or less, it is convenient for comparison with experiment to define an energy averaged cross section [11]

$$
\begin{aligned}
\vec{\sigma}^{\mathrm{DR}}\left(i, e_{c} \rightarrow d \rightarrow f\right) & =\frac{1}{\Delta e_{c}} \int \sigma^{\mathrm{DR}}\left(i, e_{c} \rightarrow d \rightarrow f\right) d e_{c}^{\prime} \\
& \equiv \frac{S^{\mathrm{DR}}}{\Delta e_{c}},
\end{aligned}
$$

where $e_{c}$ is the kinetic energy of the recombining electron. Obviously, $\vec{\sigma}^{\mathrm{DR}}$ depends on the choice of $\Delta e_{c}$, while $S^{\mathrm{DR}}=\Delta e_{c} \bar{\sigma}^{\mathrm{DR}}$ for the area under the DR peak is independent of $\Delta e_{c}$. The energy-averaged form is especially useful when experimental energy resolution of the electron beam is much larger than $\Gamma(d)$, and/or in describing many resonances which are present in a small energy interval. The choice for the energy bin $\Delta e_{c}$ is completely arbitrary, so long as $\Delta e_{c} \gg \Gamma(d)$. Two convenient choices are $\Delta e_{c}=W=$ the detector-window resolution, or $\Delta e_{c}=\Delta B=$ the electronbeam width. We choose in this paper

$$
\Delta e_{c}=W,
$$

because the DR data are presented with the width $W$. As will become clear below, however, the final comparison is independent of this choice, especially when $W>\Delta B$.

\section{RR CROSS SECTION}

We estimate the direct radiative recombination cross section $\sigma^{R R}$ for the processes

$$
\begin{aligned}
& e^{-}+[\mathrm{Ar}] 3 d^{n} \rightarrow[\mathrm{Ar}] 3 d^{n+1}+\hbar \omega, \\
& e^{-}+[\mathrm{Ar}] 3 d^{n} \rightarrow[\mathrm{Ar}] 3 d^{n} 4 l+\hbar \omega,
\end{aligned}
$$

where $n=4$ for the $\mathrm{Ba}^{34+}$ ion and $n=3$ for the $\mathrm{Ba}^{35+}$ ion. The direct recombination cross section defined for capture (8) into a completely empty subshell $n l$ is given by $[13,14]$

$$
\begin{aligned}
\sigma_{n l}^{(0) \mathrm{RR}}= & \frac{16}{3 \sqrt{2} \alpha^{2}}\left(\frac{\hbar \omega}{m c^{2}}\right)^{3}\left(\frac{m c^{2}}{e_{c}}\right)^{1 / 2}\left[l\left(R_{n l}^{e_{c} l-1}\right)^{2}+(l+1)\right. \\
& \left.\times\left(R_{n l}^{e_{c} l+1}\right)^{2}\right]\left(\pi a_{0}^{2}\right)
\end{aligned}
$$

where $\alpha=e^{2} / \hbar c$ and $e_{c}$ is the energy of the continuum electron. The radial integrals in (9) are defined as $R_{n l}^{e_{c} l^{\prime}}$ $=\int_{0}^{\infty} R_{e_{c} l^{\prime}} R_{n l} r^{3} d r$, where $R_{n l}$ and $R_{e_{c} l^{\prime}}$ are the bound state and continuum radial wave functions, respectively, and $R_{e_{c} l^{\prime}}$ is here momentum normalized [13]. For capture (7) into a partially filled shell, the RR cross section is reduced simply as

$$
\sigma_{n l}^{\mathrm{RR}}=\frac{h_{f}}{2\left(2 l_{f}+1\right)} \sigma_{n l}^{(0) \mathrm{RR}},
$$

where $h_{f}$ is the number of holes in the subshell $n l_{f}$ before the radiative recombination transition and $l_{f}$ is the orbital angular momentum quantum number. The bound-state and continuum-wave functions are calculated as in the DR case described above. Alternatively, we may also obtain the $\sigma_{n l}^{\mathrm{RR}}$ using the scaled Coulomb formula given in Ref. [9], with $Z_{\text {eff }}=\left(Z_{C}+Z_{I}\right) / 2 \sim 45$, where $Z_{C}$ is the nuclear core charge of the target ion and $Z_{I}$ is the degree of ionization of the target ion before capture. For sufficiently large $n$, the RR crosssection scales as $1 / n^{3}$, as can be seen from Kramer's formula [15],

$$
\sigma_{n}^{\mathrm{Kramer}}\left(\pi a_{0}^{2}\right)=\frac{8 \pi}{3 \sqrt{3}} \frac{\alpha_{0}^{3}}{\bar{\nu}^{3}} \frac{Z_{\mathrm{eff}}^{4}}{e_{c}\left(e_{c}+\frac{Z_{\mathrm{eff}}^{2}}{2 \vec{\nu}^{2}}\right)},
$$

where $\bar{\nu}$ is the average effective principal quantum number given by $\bar{\nu}=n-\mu$, and $\mu$ is the quantum defect. In general, the relativistic and multipole contributions are known [13] to approximately cancel each other, so that the simple nonrelativistic dipole approximation (9) seems to provide a reliable estimate.

While the DR cross section described above is peaked at a particular resonance energy, the nonresonant RR cross section is continuous and slowly varying with the beam energy. Therefore for comparison with experiment the RR cross section is integrated over an energy bin width equal to the width of the experimental electron-beam $\Delta B$ as

$$
S^{\mathrm{RR}}=\int_{\Delta B} \sigma^{\mathrm{RR}} d e_{c} \simeq \Delta B \bar{\sigma}^{\mathrm{RR}},
$$

where it is assumed that $\Delta B<W$. In the opposite case of $W<\Delta B$, the $\Delta B$ in (12) is to be replaced by $W$.

\section{RESULTS}

The spectrum shown in Fig. 1 contains all the $\mathrm{x}$ rays emitted by both the RR and DR processes for an electronbeam energy of $2.35 \mathrm{keV}$ (with strong attenuation below 2 $\mathrm{keV}$ due to absorption in the beryllium windows). Since the absolute $\mathrm{x}$ ray intensities depend on, among other factors, the trapped ion density and the overlap between the electron beam and the ions, which are not known, we focus on only 
TABLE I. The autoionization and radiative rates for the resonance state $1 s^{2} 2 s^{2} 2 p^{5} 3 s^{2} 3 p^{6} 3 d^{5}$ of the $\mathrm{Ba}^{35+}$ ion are presented in units of $(1 / \mathrm{sec})$ in the AMA coupling scheme. Brackets indicate powers of 10 .

\begin{tabular}{lcccccc}
\hline \hline$i$ & $e_{c}(\mathrm{Ry})$ & $l_{c}$ & $A_{a}\left(d \rightarrow i, l_{c}\right)$ & $f$ & $A_{r}(d \rightarrow f)$ & $\hbar \omega(\mathrm{Ry})$ \\
\hline$[\mathrm{Ar}] 3 d^{3}$ & 172 & 1 & $0.310[+14]$ & {$[\mathrm{Ar}] 3 d^{4}$} & $0.259[+15]$ & 340 \\
& & 3 & $0.418[+15]$ & {$[\mathrm{Ar}] 3 s 3 d^{5}$} & $0.136[+14]$ & 315 \\
{$[\mathrm{Ne}] 3 p^{6} 3 d^{5}$} & 131 & 1 & $0.547[+13]$ & & \\
{$[\mathrm{Ne}] 3 s 3 p^{5} 3 d^{5}$} & 141 & 0 & $0.126[+15]$ & & \\
& & 2 & $0.341[+13]$ & & \\
{$[\mathrm{Ne}] 3 s 3 p^{6} 3 d^{4}$} & 156 & 1 & $0.146[+13]$ & & \\
& & 3 & $0.126[+14]$ & & \\
{$[\mathrm{Ne}] 3 s^{2} 3 p^{4} 3 d^{5}$} & 151 & 1 & $0.528[+15]$ & & \\
& & 3 & $0.127[+14]$ & & & \\
{$[\mathrm{Ne}] 3 s^{2} 3 p^{5} 3 d^{4}$} & 166 & 0 & $0.468[+14]$ & & & \\
& & 2 & $0.493[+15]$ & & & \\
& & 4 & $0.401[+14]$ & & & \\
\hline \hline
\end{tabular}

their relative magnitudes. In this case, we are comparing resonant and nonresonant total cross sections, each with a unique energy dependence. Therefore $S^{\mathrm{DR}}$ of $(5)$ and $S^{\mathrm{RR}}$ of (12) are the basic theoretical quantities for comparison with experiment, since these are the quantities that the experimentalist directly measures. (It is also assumed that the x-ray detector for the $\mathrm{RR}$ and $\mathrm{DR}$ at $90^{\circ}$ does not affect the relative ratios.)

\section{A. Theoretical result}

The results of the calculation are presented in Tables I and II. The autoionization and radiative rates calculated in the AMA scheme are presented in units of $1 / \mathrm{sec}$. Reemphasizing that the choice of the energy averaging bin size $\Delta e_{c}$ is completely arbitrary [i.e., for $\left.\Delta e_{c} \gg \Gamma(d)\right]$, the DR cross section is averaged over a bin size equal to the resolution of the $\mathrm{x}$-ray detector $W$ where $\Delta e_{c}=W=(15 \pm 3) \mathrm{Ry}$. This dependence will drop out in the cross-section ratios. The AMA procedure generally overestimates $\sigma^{\mathrm{DR}}$ by approximately $20 \%-40 \%$, so that the corrected theoretical ratio should be reduced $[11,12]$ by a factor of 1.3 . Although the AMA theory is relatively simple, the adjustment factor in fact contains the details of state coupling; it is the result of many detailed calculations performed previously.

For the $\mathrm{Ba}^{35+}$ system, we find that the autoionization width of the resonance state $d=1 s^{2} 2 s^{2} 2 p^{5} 3 s^{2} 3 p^{6} 3 d^{5}$ is $\Gamma_{a}(d)=0.172 \times 10^{16} \mathrm{sec}^{-1}$, the radiative width is $\Gamma_{r}(d)=0.273 \times 10^{15} \mathrm{sec}^{-1}$, and thus the fluorescence yield is $\omega(d)=0.137$. The energy averaged total DR cross section for the $2 p \rightarrow 3 d$ excitation capture is then

$$
\bar{\sigma}_{\mathrm{Ba}^{35+}}^{\mathrm{DR}}=2.7 \times 10^{-21} \mathrm{~cm}^{2} / 1.3=2.1 \times 10^{-21} \mathrm{~cm}^{2},
$$

where both the $3 d \rightarrow 2 p$ and $3 s \rightarrow 2 p$ radiative decay contributions are included. For the $\mathrm{Ba}^{34+}$ case with $d=1 s^{2} 2 s^{2} 2 p^{5} 3 s^{2} 3 p^{6} 3 d^{6}$ we find that $\Gamma_{a}=0.203 \times 10^{16}$ $\mathrm{sec}^{-1}, \Gamma_{r}=0.318 \times 10^{15} \mathrm{sec}^{-1}, \omega=0.136$, yielding

$$
\underset{\sigma_{\mathrm{Ba}^{34+}}}{\mathrm{DR}}=1.9 \times 10^{-21} \mathrm{~cm}^{2} / 1.3=1.4 \times 10^{-21} \mathrm{~cm}^{2} .
$$

The $2 P_{1 / 2}-2 P_{3 / 2}$ splitting is on the order of $500 \mathrm{eV}$, so only the $2 P_{3 / 2}$ excitations satisfy the resonance condition. This is

TABLE II. The autoionization and radiative rates for the resonance state $1 s^{2} 2 s^{2} 2 p^{5} 3 s^{2} 3 p^{6} 3 d^{6}$ of the $\mathrm{Ba}^{34+}$ ion are presented in units of $(1 / \mathrm{sec})$ in the AMA coupling scheme. Brackets indicate powers of 10 .

\begin{tabular}{lcccccc}
\hline \hline$i$ & $e_{c}(\mathrm{Ry})$ & $l_{c}$ & $A_{a}\left(d \rightarrow i, l_{c}\right)$ & $f$ & $A_{r}(d \rightarrow f)$ & $\hbar \omega(\mathrm{Ry})$ \\
\hline$[\mathrm{Ar}] 3 d^{4}$ & 178 & 1 & $0.454[+14]$ & {$[\mathrm{Ar}] 3 d^{5}$} & $0.305[+15]$ & 339.0 \\
& & 3 & $0.615[+15]$ & {$[\mathrm{Ar}] 3 s 3 d^{6}$} & $0.133[+14]$ & 312.0 \\
{$[\mathrm{Ne}] 3 p^{6} 3 d^{6}$} & 135 & 1 & $0.538[+13]$ & & \\
{$[\mathrm{Ne}] 3 s 3 p^{5} 3 d^{6}$} & 145 & 0 & $0.126[+15]$ & & \\
& & 2 & $0.325[+13]$ & & \\
{$[\mathrm{Ne}] 3 s 3 p^{6} 3 d^{5}$} & 161 & 1 & $0.177[+13]$ & & \\
& & 3 & $0.147[+14]$ & & \\
{$[\mathrm{Ne}] 3 s^{2} 3 p^{4} 3 d^{6}$} & 155 & 1 & $0.517[+15]$ & & \\
{$[\mathrm{Ne}] 3 s^{2} 3 p^{5} 3 d^{5}$} & 172 & 3 & $0.126[+14]$ & & \\
& & 2 & $0.542[+14]$ & & \\
& & 4 & $0.576[+15]$ & & & \\
& & & & & \\
\end{tabular}


TABLE III. The direct radiative capture cross section $\sigma^{\mathrm{RR}}$ is given for capture into states $[\mathrm{Ar}] 3 d^{3} n l$ for the cases $n=3,4$, and 5 for the $\mathrm{Ba}^{35+}$ ion in $\left(\mathrm{cm}^{2}\right)$ corresponding to the experimental electron beam energy $e_{c}=173 \mathrm{Ry}$. Brackets indicate powers of 10 .

\begin{tabular}{lcc}
\hline \hline$f$ & $\hbar \omega(\mathrm{Ry})$ & $\sigma^{\mathrm{RR}}\left(\mathrm{cm}^{2}\right)$ \\
\hline$[\mathrm{Ar}] 3 d^{4}$ & 333.0 & $1.62[-22]$ \\
{$[\mathrm{Ar}] 3 d^{3} 4 s$} & 263.0 & $3.37[-23]$ \\
{$[\mathrm{Ar}] 3 d^{3} 4 p$} & 259.0 & $1.05[-22]$ \\
{$[\mathrm{Ar}] 3 d^{3} 4 d$} & 253.0 & $1.04[-22]$ \\
{$[\mathrm{Ar}] 3 d^{3} 4 f$} & 247.0 & $2.68[-23]$ \\
{$[\mathrm{Ar}] 3 d^{3} 5 s$} & 226.0 & $1.63[-23]$ \\
{$[\mathrm{Ar}] 3 d^{3} 5 p$} & 224.0 & $5.10[-23]$ \\
{$[\mathrm{Ar}] 3 d^{3} 5 d$} & 221.0 & $5.58[-23]$ \\
{$[\mathrm{Ar}] 3 d^{3} 5 f$} & 218.0 & $2.03[-23]$ \\
{$[\mathrm{Ar}] 3 d^{3} 5 g$} & 215.0 & $2.03[-24]$ \\
\hline \hline
\end{tabular}

accounted for by further reducing the DR cross sections obtained in the AMA scheme by a factor of $1 / 1.5$, as has been done above.

Tables III and IV show the direct radiative recombination (RR) cross sections along with the photon energies corresponding to the electron-beam energy of $172 \mathrm{Ry}=2.35 \mathrm{keV}$. Since the energy of the emitted $\mathrm{x}$ ray is the same in both cases, we first note that the peak $a b$ is a combination of DR $\left(2 p+l_{c} \rightarrow 3 d 3 d \rightarrow 2 p 3 d+\gamma\right)$ and RR $(n=3)$. From Tables I-IV, the theoretical cross-section ratios for the two ions are

$$
\frac{\bar{\sigma}_{\mathrm{Ba}^{35+}}^{\mathrm{DR}}}{\bar{\sigma}_{\mathrm{Ba}^{35+}}^{\mathrm{RR}}(n=3)} \sim 13,
$$

and

$$
\frac{\bar{\sigma}_{\mathrm{Ba}^{34+}}^{\mathrm{DR}}}{\bar{\sigma}_{\mathrm{Ba}^{34+}}^{\mathrm{RR}}(n=3)} \sim 11 .
$$

TABLE IV. The direct radiative capture cross section $\sigma^{R R}$ is given for capture into states [Ar] $3 d^{4} n l$ for the cases $n=3$ and 4 for the $\mathrm{Ba}^{34+}$ ion in $\left(\mathrm{cm}^{2}\right)$ corresponding to the experimental electron beam energy $e_{c}=173 \mathrm{Ry}$. Brackets indicate powers of 10 .

\begin{tabular}{lcc}
\hline \hline$f$ & $\hbar \omega(\mathrm{Ry})$ & $\sigma^{\mathrm{RR}}\left(\mathrm{cm}^{2}\right)$ \\
\hline$[\mathrm{Ar}] 3 d^{5}$ & 326.0 & $1.40[-22]$ \\
{$[\mathrm{Ar}] 3 d^{4} 4 s$} & 259.0 & $3.24[-23]$ \\
{$[\mathrm{Ar}] 3 d^{4} 4 p$} & 255.0 & $1.01[-22]$ \\
{$[\mathrm{Ar}] 3 d^{4} 4 d$} & 249.0 & $1.02[-22]$ \\
{$[\mathrm{Ar}] 3 d^{4} 4 f$} & 243.0 & $2.55[-23]$ \\
\hline \hline
\end{tabular}

Therefore the contribution of about $8 \%$ to the $a b$ peak from direct radiative recombination to the $n=3$ level, $(\mathrm{RR}, n=3)$, must be subtracted before comparing with the theory.

Next, we examine the relative size of the two DR peaks $a b$ and $a^{\prime}$. They arise from the initial radiationless capture $2 p+l_{c} \rightarrow 3 d 3 d$ followed by radiative decays; either $3 d \rightarrow 2 p$ (with a photon energy $4.6 \mathrm{keV}$ for $\mathrm{Ba}^{35+}$ ); or $3 s \rightarrow 2 p$ (with a photon energy $4 \mathrm{keV}$ for $\mathrm{Ba}^{35+}$ ). Similar decays occur for the $\mathrm{Ba}^{34+}$ ion. From Tables I and II, the relative probability for these two branches is

$$
A_{r}(3 d \rightarrow 2 p) / A_{r}(3 s \rightarrow 2 p) \simeq 19
$$

This is consistent with the ratio seen in Fig. 1, where the two DR peaks, $a \equiv a b-b 3$ and $a^{\prime}$, have an intensity ratio of about 16 .

In order to compare the resonant DR process with the nonresonant RR process, we adopt the energy integrated cross-section $S$. For the DR case, we have

$$
S^{\mathrm{DR}} \equiv \bar{\sigma}^{\mathrm{DR}} W
$$

which is independent of $W$, the detector resolution. For the RR case, assuming $W>\Delta B$,

$$
S^{\mathrm{RR}} \equiv \bar{\sigma}^{\mathrm{RR}} \Delta B
$$

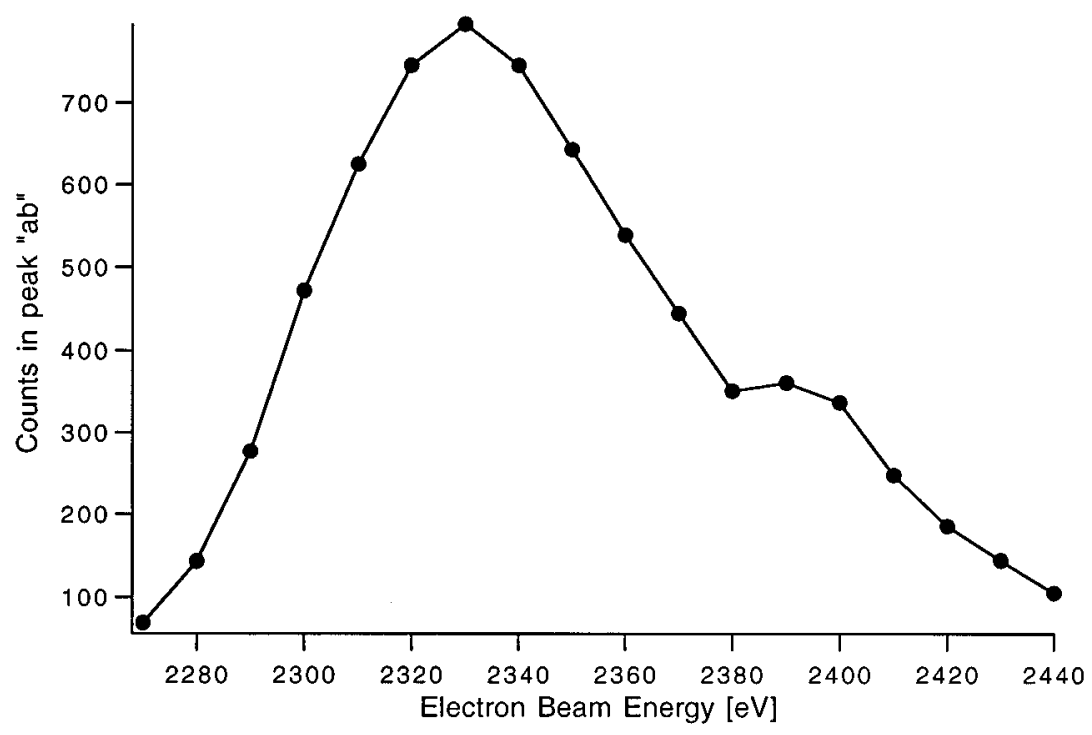

FIG. 3. Sc-like barium spectra of the DR peak $a b$ taken at electron-beam energies ranging from $2270 \mathrm{eV}$ to $2440 \mathrm{eV}$ in steps of $10 \mathrm{eV}$. The graph clearly shows the $\mathrm{Ba}^{35+}-\mathrm{Ba}^{34+}$ DR separation. 
TABLE V. The ratio of the DR and RR cross sections $\eta$ are given corresponding to capture into $4 l$ levels for the $\mathrm{Ba}^{35+}$ and $\mathrm{Ba}^{34+}$ systems.

\begin{tabular}{lc}
\hline \hline & $\mathrm{Ba}^{35+}$ \\
\hline$\eta_{\text {theory }}^{\text {AMA }}(3 d \rightarrow 2 p)$ & \\
$\eta_{\text {theory }}^{\text {AMA }}(3 s \rightarrow 2 p)$ & 0.7 \\
$\eta_{\text {theory }}^{\text {corrected }}(3 d \rightarrow 2 p)$ & $7 \pm 1$ \\
$\eta_{\text {theory }}^{\text {corrected }}(3 s \rightarrow 2 p)$ & $0.4 \pm 0.1$ \\
$\eta_{\text {theory }}^{\text {AMA }}(3 d \rightarrow 2 p)$ & $\mathrm{Ba}^{34+}$ \\
$\eta_{\text {theory }}^{\text {AMA }}(3 s \rightarrow 2 p)$ & \\
$\eta_{\text {theory }}^{\text {corrected }}(3 d \rightarrow 2 p)$ & \\
$\eta_{\text {theory }}^{\text {corrected }}(3 s \rightarrow 2 p)$ & 0.6 \\
\hline \hline
\end{tabular}

As noted earlier, $\bar{\sigma}^{\mathrm{RR}}$ is nearly independent of $\Delta B$, so that $S^{\mathrm{RR}}$ is proportional to $\Delta B$. For the present experiment, we take $\Delta B \simeq(4 \pm 1)$ Ry. (See Sec. V D and Fig. 3.)

To facilitate comparison of the DR and RR cross sections we use the $n=4 \mathrm{RR}$ capture cross section to normalize the data. This is necessary partly because of the difficulty of determining absolute ion densities and ion-electron spatial overlap inside the EBIT. The cross-section ratios are independent of these parameters, such as the ion and electron densities. For convenience, we thus define $\eta \equiv \bar{\sigma}^{\mathrm{DR}}\left(\Delta e_{c}\right) / \bar{\sigma}^{\mathrm{RR}}$, with $\Delta e_{c}$ given by (6). Treating the $\mathrm{Ba}^{35+}$ system first, we find, for the full participation of $62 p$ electrons,

$$
\eta_{\text {theory }}^{\mathrm{AMA}}(3 d \rightarrow 2 p)=\frac{\bar{\sigma}^{\mathrm{DR}}(3 d \rightarrow 2 p)}{\sigma_{4}^{\mathrm{RR}}}=\frac{3.9 \times 10^{-21} \mathrm{~cm}^{2}}{2.7 \times 10^{-22} \mathrm{~cm}^{2}} \sim 14 \text {, }
$$

and

$$
\eta_{\text {theory }}^{\mathrm{AMA}}(3 s \rightarrow 2 p)=\frac{\vec{\sigma}^{\mathrm{DR}}(3 s \rightarrow 2 p)}{\vec{\sigma}_{4}^{\mathrm{RR}}}=\frac{2.0 \times 10^{-22} \mathrm{~cm}^{2}}{2.7 \times 10^{-22} \mathrm{~cm}^{2}} \sim 0.7 \text {. }
$$

The theory must contain the AMA correction factor of 1/1.3 and the $p_{3 / 2}$ correction of $1 / 1.5$. Thus for the $\mathrm{Ba}^{35+}$ case we finally have the theoretical result

$$
\begin{gathered}
\eta_{\text {theory }}^{\text {corrected }}(3 d \rightarrow 2 p) \sim \eta_{\text {theory }}^{\mathrm{AMA}}(3 d \rightarrow 2 p) \frac{1}{1.3} \frac{1}{1.5} \sim 7 \pm 1 \\
\eta_{\text {theory }}^{\text {corrected }}(3 s \rightarrow 2 p) \sim \eta_{\text {theory }}^{\mathrm{AMA}}(3 s \rightarrow 2 p) \frac{1}{1.3} \frac{1}{1.5} \sim 0.4 \pm 0.1 .
\end{gathered}
$$

Applying the same procedure to the $\mathrm{Ba}^{34+}$ system we obtain the result summarized in Table V. For this case we find $\quad \eta_{\text {theory }}^{\mathrm{AMA}}(3 d \rightarrow 2 p)=11, \quad \eta_{\text {theory }}^{\mathrm{AMA}}(3 s \rightarrow 2 p)=0.5$, $\eta_{\text {theory }}^{\text {corrected }}(3 d \rightarrow 2 p)=6 \pm 1$, and $\eta_{\text {theory }}^{\text {corrected }}(3 s \rightarrow 2 p)=0.3 \pm 0.1$.

\section{B. Experimental result}

Since experimentally $W>\Delta B$, the RR cross section is effectively measured in terms of $\Delta B$. Therefore, following (12), it is more convenient for comparison with experiment to define

$$
\eta^{\prime} \equiv \frac{S^{\mathrm{DR}}}{S^{\mathrm{RR}}} \simeq \frac{S^{\mathrm{DR}}}{\Delta B \bar{\sigma}^{\mathrm{RR}}} \simeq \frac{\bar{\sigma}^{\mathrm{DR}}(W)}{\bar{\sigma}^{\mathrm{RR}}} \frac{W}{\Delta B} \simeq \eta \frac{W}{\Delta B} .
$$

Obviously, the $W$ dependence of $\eta$ drops out in $\eta^{\prime}$, since $\vec{\sigma}^{\mathrm{DR}}(W) W$ is independent of $W$.

We should make two further points before the comparison is made. First, for $\mathrm{Ba}^{34+}$ and $\mathrm{Ba}^{35+}$, the $\mathrm{RR} \times$ rays are only separated in energy by about $80 \mathrm{eV}$, which is well within $W$, so that $\mathrm{x}$ rays from both $\mathrm{Ba}^{34+}$ and $\mathrm{Ba}^{35+}$ are collected. Second, the experimentally derived DR cross sections are very much dependent on the relative abundances of the $\mathrm{Ba}^{35+}$ and $\mathrm{Ba}^{34+}$. The DR resonance energy difference for the two charge states is also $\sim 80 \mathrm{eV}$, which is outside the electron beam width $\Delta B$. Therefore, when $e_{c}$ is fixed at the DR resonance for the $\mathrm{Ba}^{35+}$ ion, no $\mathrm{DR}$ is allowed for the $\mathrm{Ba}^{34+}$ target ion (see Sec.V D.) The theoretical $\eta^{\prime}$ should be further multiplied by $P$, the ratio of the $\mathrm{Ba}^{35+}$ ion density to the sum of the $\mathrm{Ba}^{34+}$ and $\mathrm{Ba}^{35+}$ ion densities before comparing with experiment. Thus

$$
\eta^{\prime}=\frac{\bar{\sigma}^{\mathrm{DR}}(W)}{\bar{\sigma}^{\mathrm{RR}}}\left(\frac{W}{\Delta B} P\right)
$$

For $P \simeq 1 / 3$, obtained by combining the results of the numerical simulation [8] for the charge state distribution and an intermediate value of $\beta=0.6$, we have

$$
\frac{W}{\Delta B} P \simeq \frac{200 \mathrm{eV}}{45 \mathrm{eV}} \times 1 / 3 \simeq 1.5 \pm 0.4
$$

and thus $\eta^{\prime} \simeq \eta$ in the present experiment. (For comments on the $45 \mathrm{eV}$ beam width see Sec. V D.) As our assumption for $\beta$ varies over the full range of physically allowed values, corresponding to the DR cross sections varying from the extremes of zero to infinity, the value listed in (23) remains between 2.6 and 0 , respectively. Our estimates of uncertainty on (23) reflect a more reasonable range of allowance for the DR correction.

The experimental DR $(3 d \rightarrow 2 p)$ cross section is extracted from the data by first subtracting the $\operatorname{RR}(n=3)$ contribution. To eliminate the dependence on the ionic and electronic densities in this subtraction, we set

$$
\begin{aligned}
& \sigma_{\text {exp }}^{\mathrm{DR}, \text { adjusted }}(3 d \rightarrow 2 p) \\
& \quad \simeq \sigma_{\text {exp }}^{\mathrm{DR}}(3 d \rightarrow 2 p)-\frac{\sigma_{\text {exp }}^{\mathrm{RR}}(n=4)}{\sigma_{\text {theory }}^{\mathrm{RR}}(n=4)} \sigma_{\text {theory }}^{\mathrm{RR}}(n=3) .
\end{aligned}
$$

The $3 s \rightarrow 2 p$ transition corresponding to peak $a^{\prime}$ need not be adjusted. From the fit of the experimental data, we can directly obtain only $\eta^{\prime}$. The relative magnitudes of the DR and RR peaks $(a b$ and $b 4)$ and $\left(a^{\prime}\right.$ and $\left.b 4\right)$ are then given by 


$$
\begin{gathered}
\eta_{\exp }^{\prime}(3 d \rightarrow 2 p) \simeq 11 \pm 3, \\
\eta_{\exp }^{\prime}(3 s \rightarrow 2 p) \simeq 0.8 \pm 0.2 .
\end{gathered}
$$

Hence, to compare with (20) the quantities in (25) are converted to $\eta$ using (21) as

$$
\eta_{\exp }=\eta^{\prime} \times\left(\frac{\Delta B}{W P}\right) .
$$

Comparing (26) with (20) we find for the $\mathrm{Ba}^{35+}$ case

$$
\begin{gathered}
\eta_{\exp }(3 d \rightarrow 2 p)=7 \pm 3, \\
\eta_{\exp }(3 s \rightarrow 2 p)=0.5 \pm 2
\end{gathered}
$$

and the theoretical peak ratios are found to be in excellent agreement with the experimentally observed values.

\section{C. $\mathbf{R} \mathbf{R}$ ratios}

We also compare the relative magnitudes of the RR peaks $\xi$. The theory predicts (see Table III) for $\mathrm{Ba}^{35+}$

$$
\begin{aligned}
& \xi(4,5)_{\text {theory }}=\frac{\sigma_{5}^{\mathrm{RR}}}{\sigma_{4}^{\mathrm{RR}}}=0.54 \quad(0.51), \\
& \xi(4,6)_{\text {theory }}=\frac{\sigma_{6}^{\mathrm{RR}}}{\sigma_{4}^{\mathrm{RR}}}=0.31 \quad(0.30),
\end{aligned}
$$

and

$$
\xi(5,6)_{\text {theory }}=\frac{\sigma_{6}^{\mathrm{RR}}}{\sigma_{5}^{\mathrm{RR}}}=0.58 \quad(0.58),
$$

where the numbers in parentheses are the ratios expected from the $n^{-3}$ scaling behavior of $\sigma^{\mathrm{RR}}(n)$. The $\xi$ 's for $\mathrm{Ba}^{34+}$ (from Table IV) are similar to the above, (28). Clearly, the calculated ratios (which were done in the distorted-wave approximation) are consistent with the $n^{-3}$ scaling. This is also the case in (11) when $e_{c} \gg\left(Z_{\text {eff }}^{2} / 2 \vec{\nu}^{2}\right)$. The experiment gives

$$
\xi(4,5)_{\exp } \sim 0.56
$$

which is quite close to the theoretical value of 0.52 obtained from (26), after averaging the weighted contributions from the two charge states.

\section{Electron energy dependence of the DR peak}

The DR part of peak $a b$ [which contains both the DR and $\mathrm{RR}(n=3)$ contributions] may further be analyzed by varying the electron beam energy. Figure 3 shows two peaks corresponding to the DR contribution from $\mathrm{Ba}^{35+}$ and $\mathrm{Ba}^{34+}$, respectively. The RR $(n=3)$ contribution should be an approximately constant background on the order of $1 / 20$ of the large peak value, as shown in (15). Thus, in Fig. 3, the RR count should be on the order of 35 . The figure shows an upper bound on the electron-beam width of $\Delta B \simeq 5 \mathrm{Ry} \simeq 65$ $\mathrm{eV}$ (FWHM). The total width of the line is made up by the fine-structure splitting of the $3 d$ level into $j=3 / 2$ and $j=5 / 2$ levels and the electron-beam energy width. The $3 d$ finestructure splitting can be calculated to be around $20 \mathrm{eV}$

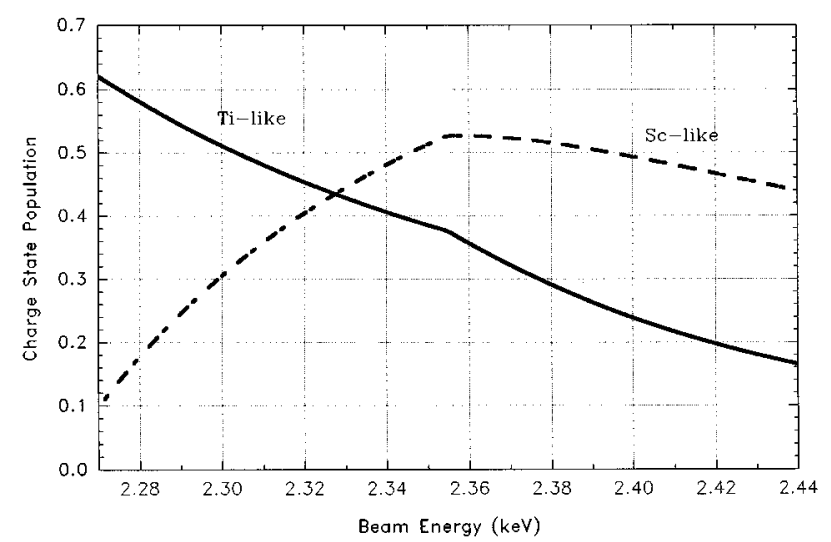

FIG. 4. Relative charge state populations of scandiumlike $\mathrm{Ba}^{35+}$ and titaniumlike $\mathrm{Ba}^{34+}$ ions in the EBIT as a function of the kinetic energy of the electron beam.

which gives a value of about $45 \mathrm{eV}$ for the beam energy width. This is in accordance with our earlier estimate for the width to be between $30 \mathrm{eV}$ and $50 \mathrm{eV}$. We used the above determined $45 \mathrm{eV}$ beam energy width throughout our analysis. The large peak in Fig. 3 centered around $e_{c} \simeq 2330 \mathrm{eV}$ corresponds to the DR process for the $\mathrm{Ba}^{35+}$ target ion, while the small peak around $2400 \mathrm{eV}$ should be the contribution from $\mathrm{Ba}^{34+}$. The reason for the reduced count rate for the second peak is the change of the charge state balance by changing the beam energy. Figure 4 shows the dependence of the $\mathrm{Ba}^{35+}$ and $\mathrm{Ba}^{34+}$ charge state populations on the electron-beam energy using the results of our model calculations (Ref. [8]). To be able to compare the experimental DR ratios with theory, one has to normalize the peak intensities to the same number of ions in the particular charge state the resonance corresponds to. This can be done using the populations of the corresponding charge states as shown in Fig. 4. The ratio of the relative population of the $\mathrm{Ba}^{35+}$ ions at 2.33 $\mathrm{keV}$ and the $\mathrm{Ba}^{34+}$ ions at $2.39 \mathrm{keV}$ is $44 \% / 26 \%=1.69$. Since this ratio does not include the effect of DR itself on the charge state balance the value given here may be biased by the fact that the DR process removes ions from the particular charge state in which they are resonant. Multiplying the theoretical DR cross-section ratio with this number our estimate for the intensity ratio is

$$
\frac{\bar{\sigma}^{\mathrm{DR}}(35+)}{\bar{\sigma}^{\mathrm{DR}}(34+)} 1.69=1.3 \times 1.69=2.2 .
$$

This is very good agreement with the experimentally observed peak intensities shown on Fig. 3. We note that the changing charge state balance complicates the analysis of the results in all the cases when the experiment requires the change of the electron-beam energy. This is the situation, e.g., when one studies resonant processes just as in our case. Since DR removes ions from the charge state in which they are resonant, there are fewer ions in the appropriate charge state at the peak centroid than in the tails. Thus, the estimate of the DR cross section obtained by using the integral of the peak is biased low, and the estimate of the width is biased high. These biases are not as important in obtaining the ratios of the two DR resonances, however. To resolve this problem in future experiments we plan to apply a transient operation 
technique. In this method one optimizes for a certain charge state balance at a given beam energy then transiently changes the beam energy to a different value where the observation is made. By choosing the off-steady-state time sufficiently short one can make sure that the number of ions in a given charge state is always the same independently of the transient energy. Alternatively, rather than the beam flipping procedure described above, the beam ramping technique may also be used.

\section{DISCUSSION}

We have presented recent experimental data on $R R$ and DR processes in $\mathrm{Ba}^{35+}$ and $\mathrm{Ba}^{34+}$ ions obtained with the EBIT at NIST, as well as a theoretical analysis. The x-ray peak $(a b)$, corresponding to the DR $2 p \rightarrow 3 d$ excitation followed by the $3 d \rightarrow 2 p$ radiative decay, has been compared to the $n=4 \mathrm{RR}$ x-ray peak $b 4$. Note that the energy of the DR $x$ ray is twice that of the incident electron kinetic energy. In addition, the smaller peak $a^{\prime}$ at $4 \mathrm{keV}$ has been shown to be consistent with the $a b$ peak and with $b 4$. In all cases, good agreement is obtained to within $\pm 20 \%$, after slight but justifiable adjustments are made to both the theoretical and experimental values. The present analysis confirms our earlier experience with the AMA procedure that, for complex openshell ions such as $\mathrm{Ba}^{35+, 34+}$, and for $W$ and $\Delta B$ large, the method works well in describing the dominant transitions. As the experimental resolution improves, more refined treatment should be necessary. Comparisons of the RR peaks relative to each other was not possible for $n>5$ due to the uncertainty in isolating their contributions from the data. However, the plateau around $2.6 \mathrm{keV}$ is probably due to the RR contribution from $n>5$. Thus all the significant features of the $\mathrm{x}$-ray spectrum above $2.4 \mathrm{keV}$ have been satisfactorily explained.

The $\mathrm{x}$-ray energies for the $\mathrm{DR}$ processes differ by $\sim 20-30 \mathrm{eV}$ for the $\mathrm{Ba}^{35+}$ and $\mathrm{Ba}^{34+}$ target ions, while $e_{c}$ for the two ions differ roughly by $80 \mathrm{eV}$. Therefore, within the resolution of the electron beam energy $\Delta B \leqslant 50 \mathrm{eV}$, it is impossible for both ions to be involved in the DR process at the same time. On the other hand, the RR peaks can have contributions from both charge states, as the detector resolution is $W \simeq 200 \mathrm{eV}$, much larger than the $80 \mathrm{eV}$ difference in the $\mathrm{x}$ rays from two different ions, for each $n$. Therefore part of the broadening of the peaks may be due to the presence of two or more charge states in the neighborhood of $\mathrm{Ba}^{35+}$. We emphasize that under the experimental conditions $W>\Delta B \gg \Gamma(d)$ the RR x-ray counts were detected from both ions $\mathrm{Ba}^{35+, 34+}$ with a band width of $\Delta B$, while the DR counts were from the $\mathrm{Ba}^{35+}$ only. Therefore, we except the spectral shapes to change as $W$ and $\Delta B$, and their ratio, are altered.

The final broad peak at the $\mathrm{x}$-ray energies $\hbar \omega<2 \mathrm{keV}$ is currently being examined theoretically [16]. This peak is produced mainly by collisional excitation fluorescence along with the cascade contribution from the primary RR process discussed above, and by Bremsstrahlung. In addition, the DR processes contribute which involve the $M$-shell electron excitations to high Rydberg levels $(n>6)$ accompanied by capture to these levels. Preliminary estimates indicate that the individual cross sections for such $M$-shell DR processes are roughly 100 times smaller than the $2 p$ excitation DR considered above. This is to be expected from the approximate $n^{-6}$ scaling. There are very many available intermediate resonance states to be included, however, with the angular momenta of the levels extending to large values $l \leqslant 10$. The explicit calculation of the $M$-shell DR is difficult and time consuming, even in the AMA procedure. A direct attempt is being made [16], employing the same procedure as in the $L$-shell DR, while a simpler alternate theoretical procedure is being developed. We also conjecture that at least some part of this low-energy $\mathrm{x}$-ray peak is the result of a recombination effect [Radiative DR(RDR)] proposed recently [17], in which the excitation capture takes place simultaneously with an x-ray emission. Such a process is DR-like, but nonresonant, and should be important when DR is weak or forbidden. Previous estimates suggest that the RDR contribution can be a few tens of a percent of DR, and can compete with RR processes as well.

Further experimental study of the DR lines in $\mathrm{Ba}^{34+}$ and $\mathrm{Ba}^{35+}$ is being planned, employing a transient technique which prevents the DR resonance from altering the charge state balance. By repeatedly switching the electron-beam energy on to resonance for approximately $10 \mathrm{~ms}$ and then off resonance for approximately $50 \mathrm{~ms}$, the charge balance may remain approximately steady at its off-resonance value. This would increase the $\mathrm{Ba}^{35+}$ ion fraction $P$ decrease its uncertainty, and simplify the analysis.

This relatively simple EBIT experiment has yielded a surprisingly diverse amount of information, as demonstrated in this report. In addition, it would be very interesting to extend the $\mathrm{x}$-ray measurement below the $2 \mathrm{keV}$ limit, where a rich variety of physical processes are expected.

\section{ACKNOWLEDGMENTS}

The theoretical part of the work reported here was supported in part by a DOE Grant.
[1] D. DeWitt, D. Schneider, M. H. Chen, M. B. Schneider, D. Church, G. Weinberg, and M. Sakurai, Phys. Rev. A 47, R1597 (1993).

[2] D. Knapp, P. Beiersdorfer, M. H. Chen, J. Scofield, and D. Schneider, Phys. Rev. Lett. 74, 54 (1995).

[3] G. Kilgus, D. Habs, D. Schwalm, A. Wolf, N. R. Badnell, and A. Müller, Phys. Rev. A 46, 5730 (1992); and J. Linkemann, J. Kenntner, A. Müller, A. Wolf, D. Habs, D. Schwalm, W.
Spies, A. Frank, A. Liedtke, G. Hoffmann, E. Salzborn, N. R. Badnell, and M. S. Pindzola, Nucl. Instrum. Methods Phys. Res. B 98, 154 (1995).

[4] Y.-K. Kim (private communication).

[5] A. Levine, R. E. Marrs, J. R. Henderson, D. A. Knapp, and M. Schneider, Phys. Scr. T22, 157 (1988); R. E. Marrs, C. Bennet, M. H. Chen, T. Cowan, D. Dietrich, J. R. Henderson, D. A. Knapp, M. A. Levine, K. J. Reed, M. B. Schneider, and J. H. 
Scofield, J. Phys. (Paris) 50, C1-445 (1989); R. E. Marrs, Comments At. Mol. Phys. 27, 57 (1991); J. D. Gillaspy, J. R. Roberts, C. M. Brown, and U. Feldman, in VIth International Conference on the Physics of Highly Charged Ions, edited by P. Richard, M. Stockli, C. L. Cocke, and C. D. Lin (AIP, New York, 1993), p. 682.

[6] Assuming a uniform charge density within the beam and then applying Gauss's law gives the correct answer to within $5 \%$.

[7] C. A. Morgan, F. G. Serpa, E. Takács, E. S. Meyer, J. D. Gillaspy, J. Sugar, J. R. Roberts, C. M. Brown, and U. Feldman, Phys. Rev. Lett. 74, 1716 (1995).

[8] B. M. Penetrante, J. N. Bardsley, D. deWitt, M. W. Clark, and D. Schneider, Phys. Rev. A 43, 4861 (1991); We thank H. Margolis for an improved C language version of B. M. Penetrante's original Fortran code, and D. Knapp for a similar but simpler independently written code.
[9] J. Gau and Y. Hahn, J. Quant. Spectrosc. Radiat. Transfer 23, 121 (1980); Y. Hahn, Phys. Rev. A 12, 895 (1975).

[10] C. Froese-Fisher, The Hartree-Fock Method for Atoms (Wiley, New York, 1977).

[11] Y. Hahn, Advances in Atomic and Molecular Physics, Vol. 21, edited by D. R. Bates and B. Bederson (Academic, New York, 1985), p. 123; Y. Hahn and K. J. LaGattuta, Phys. Rep. 166(4), 195 (1988), particularly p. 212.

[12] D. J. McLaughlin and Y. Hahn, J. Quant. Spectrosc. Radiat. Transfer 28, 343 (1982).

[13] Y. Hahn and D. W. Rule, J. Phys. B 10, 2689 (1977).

[14] D. J. McLaughlin and Y. Hahn, Phys. Rev. A 43, 1313 (1991).

[15] H. A. Kramer, Philos. Mag. 46, 836 (1923).

[16] D. McLaughlin and Y. Hahn (unpublished).

[17] Y. Hahn, Z. Phys. D 33, 247 (1995). 